\title{
HACIA UNA (POÉTICA DE LA) RELACIÓN TRANSANDINA: DE ARCHIPIÉLAGOS CARIBEÑOS Y ANDINOS O ÉDOUARD GLISSANT VS. JOHN V. MURRA
}

\author{
POR \\ Marco Thomas Bosshard \\ Europa-Universität Flensburg
}

\section{INTRODUCCIÓN: LA FORTUNA CONCEPTUAL DE LOS ARCHIPIÉLAGOS}

La imagen del archipiélago y las metáforas de la insularidad vinculadas a ella aparecen en el discurso caribeño a partir de los años 1980, ante todo en las obras de Édouard Glissant y Antonio Benítez Rojo (La isla que se repite). Sin embargo, parece que hasta hoy no han perdido nada de su atractivo. Al contrario, el paradigma del archipiélago se está expandiendo aún más, como demuestra un volumen colectivo publicado recientemente bajo el título Worldwide. Archipels de la mondialisation. Archipiélagos de la globalización (Ette y Müller). Allí, la teoría (o, tal vez mejor: las teorías) del archipiélago se están aplicando también fuera del Caribe, por ejemplo en el caso de las islas de Mauricio, Madagascar, Oceanía e incluso, en tierra firme, en los casos de Québec y Brasil. Los contribuyentes provienen en su mayoría del área francófona y llegan a conclusiones diferentes. Pero al mismo tiempo, muchos de los artículos que se encuentran allí reunidos se refieren de alguna manera al escritor y ensayista martiniqués Édouard Glissant, muerto en 2011: un hecho que enfatiza el impacto de su obra.

No obstante, en todo este debate sobre el archipiélago globalizado, se olvida completamente otra región latinoamericana, donde el concepto del archipiélago no solamente constituye una pauta fundamental para la descripción de ciertas estructuras socioeconómicas, sino también tiene su propia historia y, sobre todo, su propia fortuna -ya antes de que Glissant planteara sus teorías-. Nos referimos al área andina y a las propuestas del antropólogo estadounidense, de origen ucrainiano, John Victor Murra, muerto en 2006.

Obviamente, el uso del concepto del archipiélago en la zona andina a primera vista puede resultar sorprendente, porque su topografía concreta no coincide con la realidad caribeña, donde describe unas condiciones geográficas inmediatas. Sin embargo, a 
continuación intentaremos echar una segunda mirada a esta coincidencia terminológica supuestamente tan contingente. Por un lado, releeremos y resumiremos a Glissant; por el otro, haremos lo mismo con Murra (autor desconocido para la mayoría de quienes trabajan sobre Glissant), con el propósito de llegar a una comparación crítica. Por eso analizaremos, tanto en la primera parte sobre Glissant como en la segunda sobre Murra, los aspectos geográficos y socioeconómicos a la vez que los metafóricos y discursivos. Por fin, retomaremos las propuestas de críticos andinos como Zevallos Aguilar (ver "Hacia una topografía" y "Desplazamiento y transnacionalismo") y Noriega Bernuy (ver Caminan los Apus), abordando las posibilidades que nos brinda la metáfora del archipiélago para llegar a una contextualización de lo que puede significar la relación transandina en el contexto de los discursos contemporáneos sobre el paradigma -y el reto- del transnacionalismo.

\section{Édouard Glissant y el desarrollo del "PENSAMiento archipélico"}

Glissant desarrolla sus planteamientos alrededor de la pensée archipélique, el "pensamiento archipiélico", en varios pasos. ${ }^{1}$ En 1980, cuando publica Le discours antillais, la noción del pensamiento archipiélico aún no existe; a lo largo de las 800 páginas de la obra, la palabra francesa "archipel” se utiliza una sola vez. En este único pasaje, el archipiélago resulta yuxtapuesto al continente -pero todavía no en forma de una dicotomía estratégica entre pensamiento archipiélico y pensamiento continental que Glissant desarrollará más tarde en la Introduction à une Poétique du Divers y en el Traité du Tout-Monde de los años 1990. Más bien, en Le discours antillais la palabra "archipiélago" (ya que todavía no se puede hablar de un concepto) remite a circunstancias geográficas, señalando el hecho de que Glissant dé por sentado que las formas del pensamiento - para no decir de las mentalidades-resultan condicionadas por factores geográficos, geológicos y geofísicos que a su vez generan sus correspondientes estructuras agrarias y socioeconómicas. En el capítulo “À partir du paysage”, Glissant pone en escena a su isla natal, Martinica, como una especie de microcosmos que refleja la situación geográfica de toda América: así, el norte de la isla está dominado por las montañas, después hay la llanura, la Pleine, y en el sur predomina la arena. Por eso, Glissant puede sostener: "Il y a [...] continuité, de l'archipel au continent" (Le discours antillais 390). Apoyándose, por un lado, en la tipología de los pueblos americanos que propuso Darcy Ribeiro en Las Américas y la civilización varios años antes de que fuera retomada por Glissant, y por otra parte, en el modelo presentado por el jamaicano Rex Nettleford en Caribbean Cultural Identity, que intenta categorizar las poblaciones

1 Como introducción a los escritos de Glissant, ver los trabajos editados por Chevrier en el marco de un coloquio sobre Glissant o, en lengua castellana, el resumen de Mazeau de Fonseca.

Revista Iberoamericana, Vol. LXXXI, Núm. 253, Octubre-Diciembre 2015, 973-985 
étnicas en relación a sus respectivas zonas de producción agraria, Glissant equipara los pueblos testimonio de Ribeiro con la noción de "Meso-América"; esta noción, según Nettleford, abarca los pueblos americanos donde predomina el cultivo del maíz y de la papa, lo que significa que "Meso-América" no comprende solamente la Mesoamérica geográfica que se extiende desde México al istmo de Panamá, sino también los Andes. Los pueblos transplatados, en cambio, corresponden a "Euro-América", y los pueblos nuevos, finalmente, equivalen a la "Plantation America", donde predomina, conclusión obvia, la economía de la plantación. Así, toda América, que se condensa en la isla de Martinica, se define

[...] à partir de trois lieux: les hauts des Andes où la passion indienne perdure, le Mitan des plaines et des plateaux où le métissage s'accélère, la mer Caraïbe où les îles présagent. J'ai dit en introduction à ce livre que le paysage martiniquais (le Nord et le mont, la plaine du Mitan, les sables au Sud) reproduit en résumé de telles ambages. (Le discours antillais 390-391)

Se nota en esta cita que el eje vertical, que conecta las cumbres de Martinica con las playas arenosas al nivel del mar, tiene cierto peso; no obstante, Glissant se interesa casi exclusivamente por el eje horizontal que reúne las islas del archipiélago (Martinica y el resto del Caribe) en lo que llama una "multi-relación". En esto se inspiró, según manifestó en varias ocasiones, en la idea del rizoma como la definen Gilles Deleuze y Félix Guattari en Mille plateaux. Así, la cuasieliminación del eje vertical en los planteamientos de Glissant se explica también a la luz de sus referencias teóricas, porque en Deleuze y Guattari, el eje vertical resulta asociado con las jerarquías de la lógica tradicional (y occidental) de las raíces y de los árboles, mientras que en la conceptualización del contramodelo rizomático, los dos autores exaltan la extensión infinita de las llanuras con sus líneas de fuga y horizontes siempre en movimiento y, por ende, también infinitos. Glissant, a su vez, no deja lugar a dudas que el eje vertical coincide con la lógica de la raíz, y el eje horizontal con el rizoma desjerarquizado: "Je vous rappelle que la racine unique a la prétention de la profondeur et que la racine rhizomée s'étend dans l'étendue" (Introduction à une Poétique du Divers 52). ${ }^{2}$

\footnotetext{
Nos parece una simplificación ilegítima esa reducción del rizoma a lo horizontal o a lo extendido. Con respecto a los Andes, existen estructuras iguales a las del rizoma (el mallki) que los mismos informantes indígenas mencionan para describir las conexiones verticales que existen entre núcleos e islas (ver el apartado sobre Murra) de su organización social archipiélica. Así, Platt recuerda la anécdota siguiente: "Entonces, mientras diagramaba las conexiones verticales entre grupos de parentesco del valle y de la puna, me pregunté de qué manera las respectivas 'raíces' de las unidades domésticas, conectadas localmente con esos árboles y torres, podían estar conectadas también a través de los declives de la cordillera. Una posible solución al problema me fue sugerida por un conocido de Macha que me contó que mallki era el nombre quechua de 'aquellas plantas que arrojan otras plantas'. ¿Cabría imaginar los
}

Revista Iberoamericana, Vol. LXXXI, Núm. 253, Octubre-Diciembre 2015, 973-985 ISSN 0034-9631 (Impreso) 
Es exactamente aquí donde el discurso geográfico y socioeconómico que maneja Glissant se convierte en un discurso metafórico. Así como el archipiélago caribeño se extiende horizontalmente, también se proyectan en su superficie las supuestas estructuras rizomáticas del pensamiento y de la mentalidad caribeños. De esta manera, el verbo $s$ 'étendre -“dilatarse", "extenderse"- gana más y más en importancia, hasta el momento tautológico cuando "se extiende en lo extendido" ("s'étend dans l'étendue"). Geográfica y económicamente hablando, se puede relacionar lo extendido ("l'étendue") con la economía de las plantaciones extensas tan típicas en el Caribe; pero Glissant también recurre al verbo cuando habla de las estrategias de apropiación de la escritura por una sociedad como la martiniquesa que destaca por su carácter oral. A más tardar en Poétique de la Relation (1990), lo extendido se transforma en un concepto cabal, constituyendo la antítesis de lo que Glissant llama la filiación. Ésta se refiere a un principio de descendencia ramificada, pero siempre jerárquico y fijo. El emblema de la filiación es el árbol genealógico, la visualización perfecta de la raíz única occidental y patriarcal; lo extendido, en cambio, se representa a través del rizoma. Significativamente, ya en Le discours antillais, lo extendido aparece también en el contexto de las estructuras matriarcales o (mejor) matrifocales de la familia martiniquesa. ${ }^{3}$ Según Glissant, el "corps familial martiniquais" destaca por el predominio de la "tradition [...] de la lignée par les femmes, ainsi que l'organisation de la famille étendue (sœurs aînes, tantes, marraines, grand-mères)" (Le discours antillais 166). Y también en obras posteriores, como por ejemplo en Poétique de la Relation, este "style de la famille étendue" (70) matrifocal se sigue enfatizando.

Son entonces las estructuras antipatriarcales, antioccidentales, antiesenciales y rizomáticas las que le interesan a Glissant; no es el ser (l'être), sino el estar (l'étant) que lo inspira, el pensamiento nómada que incansablemente está en movimiento. En el Traité $d u$ Tout-Monde, Glissant por fin sintetiza todas estas connotaciones a través del concepto del "pensamiento archipiélico"; de nuevo, nótese en la cita siguiente el vínculo inevitable entre este pensamiento archipiélico, lo extendido y el horizonte:

La pensée archipélique convient à l'allure de nos mondes. Elle en emprunte l'ambigu, le fragile, le dérivé. Elle consent à la pratique du détour, qui n'est pas fuite ni renoncement. Elle reconnaît la portée des imaginaires de la Trace, qu'elle ratifie. Est-ce là renoncer

lazos verticales como un sistema de raíces subterráneas, como los 'estolones' de las plantas de fresa, o sea, tallos rastreros que se enraízan a una distancia de la planta madre? ¿Tenían los colonos que ‘enraizarse a sí mismos' como isleños del valle, habiendo sido enviados hacia abajo por sus grupos de parentesco asentados en la puna?” (300-301).

3 Insistimos tanto en esto, porque también Murra se había dedicado, antes de que se convirtiera definitivamente en andinista, al estudio de las estructuras de la familia caribeña; retomaremos este tema más adelante.

Revista Iberoamericana, Vol. LXXXI, Núm. 253, Octubre-Diciembre 2015, 973-985 
à se gouverner? Non, c'est s'accorder à ce qui du monde s'est diffusé en archipels précisément, ces sortes de diversités dans l'étendue, qui pourtant rallient des rives et marient des horizons. (Traité du Tout-Monde 31)

En la Introduction à une Poétique du Divers se exponen de manera más exacta las implicaciones de este "pensamiento archpiélico" y, también, las oposiciones. Lo que Glissant llama aquí pensée continental, el "pensamiento continental", asume todas las connotaciones de lo occidental, lo patriarcal y del principio de la raíz y de la descendencia que acabamos de mencionar:

Hier [...], la pensée [...] a organisé, étudié, projeté ces répercussions lentes et insensibles entre les langues -a prévu et mis en perspective idéologiquement le mouvement du monde qu'elle régentait légitimement. Aujourd'hui, cette pensée de système que j'appelle volontiers "pensée continentale" a failli à prendre en compte le non-système généralisé des cultures du monde. Une autre forme de pensée, plus intuitive, plus fragile, menacée, mais accordée au chaos-monde et à ses imprévus, se développe [...] J'appelle cette pensée une pensée "archipélique" [...] Ce que je vois aujourd'hui, c'est que les continents "s'archipélisent", du moins du point de vue d'un regard extérieur. Les Amériques s'archipélisent, elle se constituent en régions par-dessus les frontières nationales. (Introduction à une Poétique du Divers 33-43)

Por supuesto, esta última frase de Glissant abre el debate sobre el fenómeno del transnacionalismo y de la revalorización de los territorios regionales frente a los nacionales; volveremos sobre esto en los apartados finales de este artículo.

\section{John Murra: del CARIBe al archipiÉlago andino}

Si nos dejáramos seducir por el estilo sugestivo de Glissant, la búsqueda de archipiélagos en la sierra (andina) estaría condenada al fracaso desde el principio. Por más que lo estimemos, nos parece que Glissant se aferra demasiado a sus binarismos metafóricos del pensamiento archipiélico y continental, a sus dicotomías entre el ser eterno y el estar nómade. No es casual que descalifique la ontología continental justamente a través de la imagen de la montaña: "L'Être est immobile en montagne, il s'est protégé de neige et d'avalanche impénétrable" (Traité du Tout-Monde 237). Pues si enfocamos la trayectoria personal de John Murra, nos topamos con un período clave en el Caribe que le permitió a Murra -tal nuestro planteamiento- esbozar un modelo que luego aplicó, con mucho éxito, al mundo andino. Pues a fines de los años 1940, Murra pasó un tiempo considerable enseñando en la Universidad de Puerto Rico. Y aún después de su tesis doctoral de 1956 sobre The Economic Organization of the Inca State, dedicada ya a la zona andina, seguía dirigiendo y participando en varios estudios de campo en el Caribe durante el tiempo cuando era profesor en el Vassar College.

Revista Iberoamericana, Vol. LXXXI, Núm. 253, Octubre-Diciembre 2015, $973-985$ ISSN 0034-9631 (Impreso) 
Sus estudios de entonces se enfocaron en el análisis de las estructuras familiares del Caribe anglo y francófono. Como antropólogo profesional (y al contrario de Glissant que dijo en algún momento que odiaba la etnografía), Murra nos brinda las referencias exactas (los trabajos de Metraux, por ejemplo) para sustentar el fenómeno de los "matrifocal families with correspondingly marginal roles for husbands and fathers" ("Studies in Family Organization" 374) en Martinica, coincidiendo en eso con la observación de Glissant acerca de la familia extendida martiniquesa con su linaje de mujeres. Sin embargo, Murra no se contenta con eso. Inmediatamente después, Murra relativiza esta observación diferenciándola con referencia a su propio estudio de campo efectuado en Martinica: "Nevertheless, even in the short period of field work at our disposal, we were able to ascertain that family and household forms do tend to differ according to region" (375). Es muy interesante que esta diferenciación parta desde el paradigma de la verticalidad que Murra establecería, años más tarde, como categoría cabal en el ámbito de la etnografía andina. Pues también en el caso de Martinica, la mirada de Murra enfatiza el eje vertical en la topografía de la isla, porque según él, en los "Maroon communities" de la sierra martiniquesa, a menudo respaldados por clérigos europeos, es el tipo del "European father [...] who plays the propelling role", mientras que las famosas estructuras matrifocales solamente predominan abajo, es decir "in the plantation lowland of Lamentin and Basse Pointe" (376).

Por supuesto, éste no es el lugar para comentar los aportes de Murra a la etnografía caribeña; y lo es aún mucho menos, si tenemos en cuenta sus comentarios poco espectaculares acerca de los años pasados en Puerto Rico y también de las "temporadas de campo [...] en otros lugares del Caribe, especialmente Jamaica y Martinica", que resume de la manera siguiente: "Todo esto fue agradable, además de instructivo, pero todo el tiempo supe que pertenecía a los Andes" ("Entrevista con John Murra"). Sin embargo, con el fin de formular una hipótesis -por qué Murra recurre precisamente a las metáforas de la isla y del archipiélago para apoyar su análisis de las estructuras socioeconómicas en los Andes-, nos arriesgaríamos a afirmar que su "interludio en el Caribe" ("Entrevista con John Murra") nos brinda una explicación posible. Si echamos una mirada al periódico del Vassar College de aquel tiempo (ver imagen), salta a la vista el titular "The Caribbean: Peoples in Flux"; después sigue el artículo dedicado a los estudios de campo de Murra en Jamaica y Martinica. Aunque parezca ser un hecho, que el paradigma del nomadismo y de las "culturas en movimiento" está presente de manera casi arquetípica siempre que la crítica se refiere al Caribe, resulta que Murra, en tanto que nómada académico, se reorientó hacia los Andes para volverse antropólogo. Pero tal vez podemos decir que este tipo de análisis antiesencialista in flux de la comparación de culturas que Murra practicaría también al escribir sobre las culturas andinas, se formó gracias a aquellos años de aprendizaje en el Caribe.

Pues a partir de los años 1960, Murra se enfrentó en los Andes con el mismo fenómeno de diversidad étnica, en la unidad paradójica de un archipiélago, que ya

Revista Iberoamericana, Vol. LXXXI, Núm. 253, Octubre-Diciembre 2015, 973-985 
conocía en su variante caribeña. En este caso, ya no es tanto el imperio incaico lo que le interesa, sino más bien la diversidad étnica del tiempo preincaico, un fenómeno que seguía existiendo también durante el dominio de los incas. Así, es legítimo preguntarse cómo esta idea de un imperio enorme con sus estructuras necesariamente jerárquicas, que se extiende por la mitad del continente, puede resultar compatible con la idea de diversidad étnica. ¿Acaso estamos ante una constelación en la cual el pensamiento continental de Glissant convive simultáneamente con el pensamiento archipiélico? ¿Acaso en esta situación se está archipielizando el continente? Aunque uno se inclinaría a aceptar esta explicación metafóricamente tan sugestiva a primera vista, nos parece que sería erróneo identificar aquel pensamiento continental con las estructuras del antiguo Tawantinsuyu, pues el imperio incaico, habiendo además incorporado aquellas estructuras archipiélicas de culturas anteriores que Murra ha descrito, difícilmente puede ser caracterizado mediante categorías estáticas occidentales.

Como Glissant, en cuyos escritos tempranos podemos observar una analogía entre el horizonte infinito y las plantaciones infinitas del Caribe, también Murra parte de la topografía andina y de las condiciones climáticas y formas agrarias condicionadas por ella. En su famoso estudio "El control vertical de un máximo de pisos ecológicos" (1972) Murra describe la estrategia de muchas etnias andinas para aprovechar en su agricultura un máximo de zonas o niveles climáticos denominados pisos ecológicos. En eso, el eje vertical, y con él también el control vertical, es cabal vista la topografía andina, pues resulta obvio que a 3000 y 4000 metros de altura pueden cultivarse muy pocos de los productos necesarios para la vida cotidiana, de modo que las etnias locales trataban de fundar colonias agrarias en otros niveles verticales, desplazándose hacia la selva amazónica o hacia la costa pacífica, para poder brindarles a sus núcleos altiplánicos productos como guano, madera, miel, etc. Es en este contexto que aparece en "El control vertical” el término de las islas ecológicas, vinculadas con sus núcleos a través de relaciones familiares y de reciprocidad, a pesar de que se ubicaban a distancia considerable de ellos. Todas estas islas ecológicas en los distintos pisos, especializadas en el cultivo de productos típicos de la zona, formaban, junto con su núcleo, este famoso archipiélago vertical, que se caracteriza tanto por su discontinuidad espacial como por su continuidad cultural:

Estas "islas" étnicas, separadas físicamente de su núcleo pero manteniendo con él un contacto social y tráfico continuo, formaban un archipiélago, un patrón de asentamiento típicamente andino. A este tráfico, que permitía el acceso simultáneo a recursos y zonas muy distintos entre sí por una misma población, se le ha llamado "comercio", usando modelos de otras latitudes. Se le ha confundido también con migraciones estacionales o trashumancia. De hecho, en diversas partes del mundo andino, la economía colonial y después la capitalista, han reducido hoy en día los archipiélagos verticales a relaciones limitadas de trueque ritual o a intercambios estacionales. ("Los límites" 94)

Revista Iberoamericana, Vol. LXXXI, Núm. 253, Octubre-Diciembre 2015, 973-985 ISSN 0034-9631 (Impreso) 


\section{Vassar Miscellany News \\ in.}

\section{THE CARIBBEAN: PEOPLES IN FLUX ...}

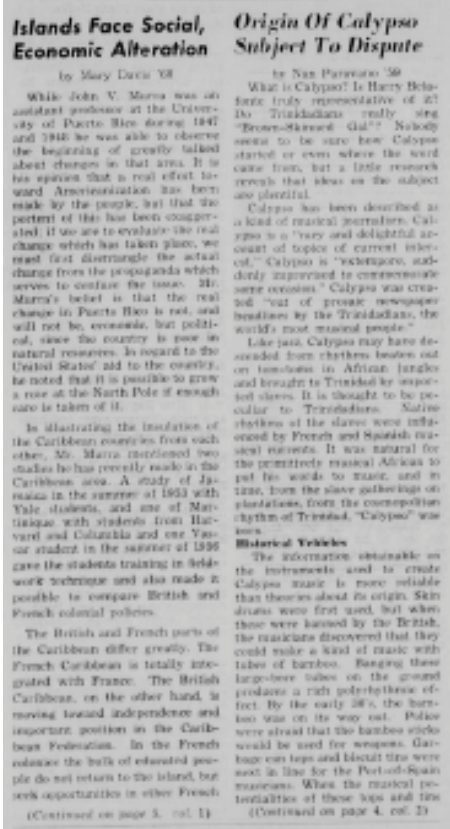

\section{Legislative Meeting . . .}

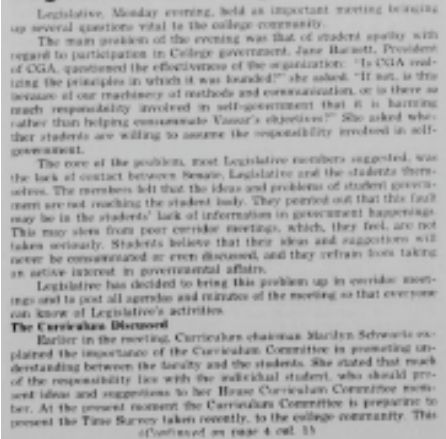

Srlinduk

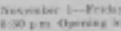

of the Podien in the Bis

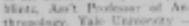

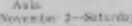

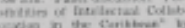

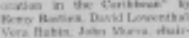

60 in Leture win ane

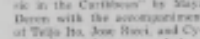

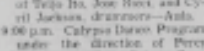

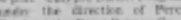

Openimg Spruber...

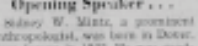

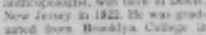
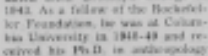

amed his 1 is

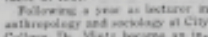
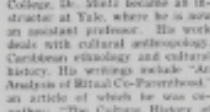

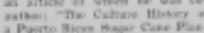

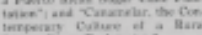

CGA Plans Series

Of Faculty Lectures

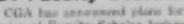

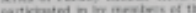

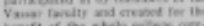
moves of the stole citho oum.

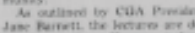

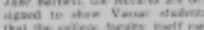

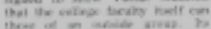

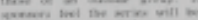

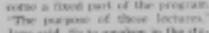

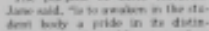

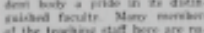

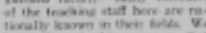
hom thend the proran of

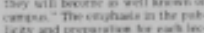

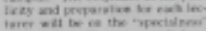

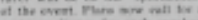

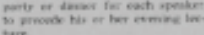

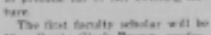
n- beily Chink Bresh polfo.

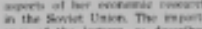

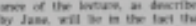

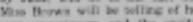

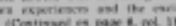

Experts View Caribbean Problems; See Need For Understanding Area

Griffin Lectures On

U.S. Foreign Poliey

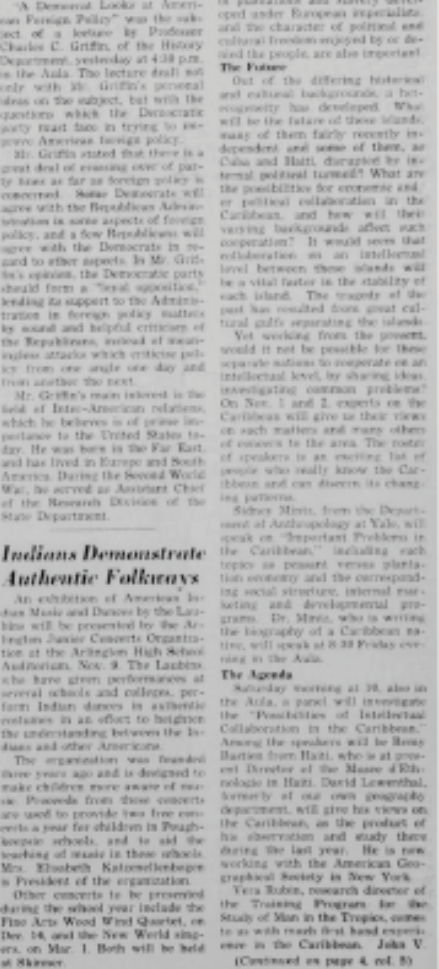

Portada de la revista Vassar Miscellany News, vol. XXXII [sic] 30 de octubre 1957.

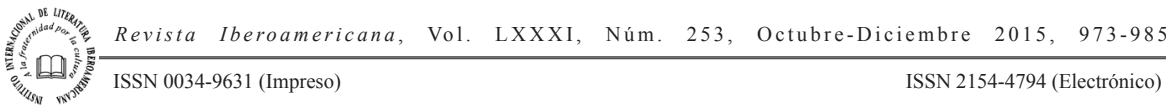


Mientras que la primera parte de la cita de Murra expresa exactamente lo que acabamos de parafrasear arriba, la segunda parte enfatiza otro aspecto cabal de los archipiélagos andinos. Murra resalta el hecho de que la economía archipiélica no equivale a las formas tradicionales de "comercio" o, incluso, de "trueque" según la percepción occidental. Más bien, se trata de una forma singular y alternativa de subsistencia a la escala de una nación entera que, no obstante, conoce un alto grado de división del trabajo; al mismo tiempo, esta comunidad no queda restringida a las fronteras territoriales, constituyendo una especie de nación transterritorial. Y Murra menciona otro aspecto más, también muy característico de estas islas que forman el archipiélago vertical:

Una característica inesperada más de las islas periféricas: con frecuencia éstas están compartidas por varios grupos altiplánicos. Hubo competencia, muchas y temporeras hegemonías por todo el nicho, pero en muchas y frecuentes situaciones, diversos núcleos se vieron obligados a compartir los recursos de una vallada, de un cocal o de un oasis durante treguas, en coexistencia tensa, pero real. (95)

Aunque el núcleo sea étnicamente homogéneo, no lo son las islas del archipiélago; por el contrario, destacan por su población multiétnica. Así, las islas ecológicas se convierten en zonas de contacto intercultural de las etnias andinas, en las que todas tratan de aprovechar la producción agraria específica de su nicho o piso ecológico, viéndose obligadas a organizar una convivencia viable sin conflictos demasiado grandes. ${ }^{4}$

El ARCHIPIÉLAGO ANDINO COMO MANIFESTACIÓN MODIFICADORA DE LOS MODELOS DEL TRANSNACIONALISMO Y COMO POÉTICA

Estas dos características del archipiélago vertical - el uso de lugares de producción lejanos del centro y la diversidad étnica de sus islas - nos brindan la base teórica de un modelo alternativo para analizar el fenómeno de comunidades transnacionales (sean éstas andinas o no). Aunque el modelo de Murra parte de las condiciones socioeconómicas específicas de los Andes durante un período pasado, en forma abstracta tiene vigencia también para la condición de migrantes tanto andinos como no andinos en la época contemporánea. ${ }^{5}$

4 Por supuesto, ha habido críticas (e incluso una autocrítica del propio Murra) de este modelo archipiélico andino que se problematizan en el artículo de Zevallos Aguilar en este volumen. Ya que nuestro objetivo no consiste tanto en criticar el modelo en sí, sino en cuestionar y abordar las metáforas (y su genealogía) que lo sustentan, renunciamos aquí a comentarios de esta índole.

5 Se trata de un modelo que Zevallos Aguilar (ver "Hacia una topografía") ya esbozó hace unos doce años y que retoma en su artículo en este volumen.

Revista Iberoamericana, Vol. LXXXI, Núm. 253, Octubre-Diciembre 2015, 973-985 ISSN 0034-9631 (Impreso) 
Si repasamos uno de los textos más citados para poder definir el fenómeno del transnacionalismo -el trabajo ya clásico de Steven Vertovec: "Conceiving and Researching Transnationalism”-, estamos ante seis características generales. Primero, Vertovec destaca una morfología social específica, determinada por la presencia de diásporas étnicas - una noción muy aceptada hoy en día que aparece también en el segundo punto que menciona Vertovec, cuando percibe el transnacionalismo como una forma de consciencia colectiva o de "diaspora consciousness". Sin embargo, habrá que poner en tela de juicio el concepto de "diáspora": más allá de la historia conceptual teñida de muchas connotaciones religiosas que implica el término, hay otras razones para preferir el concepto del archipiélago para poder definir esas nuevas formas de la morfología social transnacional. Al contrario de la palabra diáspora, que significa “dispersión”, la etimología del archipiélago resalta el mar (pelagos) no como elemento que separa sino que reúne las islas, por lo cual se subrayan las relaciones unificadoras de los grupos migratorios que habitan en territorios lejanos de sus orígenes, pero sin caer en la trampa de la exaltación de un homeland perdido que hay que recuperar algún día. Además, está presente en el modelo archipiélico según Murra (y las propuestas de Zevallos Aguilar y Noriega Bernuy basadas en él) el componente económico, que tal vez es el aspecto más importante del transnacionalismo. Pues saltándonos el tercer punto de Vertovec sobre el transnacionalismo como fenómeno de producción cultural vinculada a la hibridez, a la creolización o al sincretismo -aquí sí, Glissant y su término de "creolización" es compatible-, llegamos al cuarto aspecto del transnacionalismo que constituye la categoría económica de los flujos del capital. Como forma institucionalizada, hay que mencionar aquí las famosas empresas multi o transnacionales, aunque igual de importante en nuestro contexto resulta la economía informal de los migrantes con sus envíos de dinero a sus países de origen. Esta economía transnacional desde abajo, desde los propios migrantes que trabajan en islas dispersas por todo el mundo para sus familias que siguen viviendo en núcleos lejanos, se corresponde en algunos aspectos con la economía alternativa del archipiélago vertical andino que así llega a interferir en los flujos contemporáneos del capital. Teniendo en cuenta las condiciones precarias del capitalismo globalizado, el modelo del archipiélago contiene una contrapropuesta porque es transnacional, pero sin compartir el objetivo principal del comercio, de la ganancia y de los superávit del transnacionalismo económico. Aunque sería ingenuo creer que esta forma económica transnacional basada en la idea de la reciprocidad andina pueda reestablecerse a nivel global, no obstante nos brinda un modelo alternativo para enfrentar los retos de la globalización no como un gran mal, sino como un hecho e incluso como una necesidad. Por lo menos, no nos parece tan descabellada la idea de que la economía archipiélica pudiera conectar con ciertas iniciativas de las ONGs y de otras organizaciones sin fines de lucro que se mencionan en el catálogo de Vertovec en el quinto punto donde habla del transnacionalismo como lugar de compromiso político

Revista Iberoamericana, Vol. LXXXI, Núm. 253, Octubre-Diciembre 2015, 973-985 
a nivel internacional. Por fin, la posible doble pertenencia tanto a la isla multiétnica en territorio ajeno como al núcleo lejano de origen fomenta la nueva construcción de localidades y translocalidades, con lo cual Vertovec termina su enumeración de fenómenos transnacionales.

Aunque pueda parecer que las implicancias económicas del archipiélago andino de Murra nos hubieran hecho preferir el modelo de éste frente al modelo de Glissant, urge rescatar el aspecto poético -es decir, creativo-del cual nos habla el último y que está ausente en Murra, pues a través del pensamiento archipiélico, Glissant concibe una "Poética de la Relación" que también puede resultar sugerente para definir la relación transandina. ${ }^{6}$ Para Glissant, esa Relación (siempre con mayúscula) consiste en la concientización de que vivimos, lo queramos o no, en un mundo globalizado con todas sus estructuras hegemónicas y violentas - pero también con todos sus aspectos más agradables-. Es esta consciencia acerca de la inevitabilidad de este nuevo sistema mundial -y sobre todo, es el enfrentamiento creativo, no resignativo con él, catalizado por el pensamiento archipiélico- que genera la poética de esta Relación. La Relación abarca todo, todo el caos del Todo-Mundo, y puesto que en ella todo está en contacto con todo, resulta que "nous n'avons plus besoin, quand nous évoquons une poétique de la Relation, d'ajouter: relation entre quoi et quoi?" (Poétique de la Relation 39-40) En el punto culminante premoderno e preincaico del archipiélago vertical andino, este saber acerca de la globalización venidera que empezó con la Conquista, aún no existía, pero tal vez sí un presentimiento arquetípico. Así como la convivencia viable de las etnias sobre la base de las condiciones de producción en los distintos archipiélagos andinos puede ser descrito como un transnacionalismo avant la lettre, este mismo hecho ya prefigura la Relación universal según Glissant, donde todo(s) está(n) en contacto con todo(s). Aun si el paradigma del archipiélago económico genealógicamente se limita a un territorio particular en el continente sudamericano premoderno (por lo cual optamos nosotros por la minúscula), los conflictos, pero también la convivencia (de vez en cuando incluso exitosa) de esta relación transandina anticipan conflictos contemporáneos y -ojalá- también formas de convivencia más viables en la época globalizada actual. ${ }^{7}$ Son los artistas, poetas y pensadores, a los cuales se dedican los artículos en este volumen, quienes son los personajes que convierten estas relaciones transandinas - dadas e inevitables-en poética, brindándonos puntos de orientación en el caos del mundo y, también, de vez en cuando, desorientaciones muy necesarias en el orden sospechoso de cada día.

${ }^{6}$ En este punto son también sugerentes las reflexiones sobre constelaciones y configuraciones transandinas, propuestas por Pablo Valdivia Orozco en este volumen.

7 Sobre las relaciones transandinas y la convivencia en la literatura, ver la colaboración de Ottmar Ette en este volumen.

Revista Iberoamericana, Vol. LXXXI, Núm. 253, Octubre-Diciembre 2015, 973-985 ISSN 0034-9631 (Impreso) 
BiBLIOGRAFÍA

Benítez Rojo, Antonio. La isla que se repite: El Caribe y la perspectiva posmoderna. Hanover, N.H.: Ediciones del Norte: 1989.

Chevrier, Jacques, ed. Actes du colloque international de la Sorbonne. 1998. París: Presses de l'Université de Paris-Sorbonne, 1999.

Deleuze, Gilles y Félix Guattari. Mille Plateaux. París: Les Editions de Minuit, 2006. "Entrevista con John Murra". Boletín del Museo de Oro 17. 1986. <http://www. banrepcultural.org/blaavirtual/publicacionesbanrep/bolmuseo/1986/bol17/boc3. htm>. 1 marzo 2013.

Ette, Ottmar y Gesine Müller, eds. Worldwide: Archipels de la mondialisation. Archipiélagos de la globalización. Madrid/ Fráncfort: Iberoamericana/Vervuert, 2012.

Glissant, Edouard. Le discours antillais. 1981. París: Gallimard, 1997. Introduction à une Poétique du Divers. París: Gallimard, 1996. Poétique de la Relation. Poétique III. París: Gallimard, 1990. Traité du Tout-Monde. Poétique IV. París: Gallimard, 1997.

Mazeau de Fonseca, Patricia. "Algunas reflexiones sobre la Poética de la Relación de Édouard Glissant”. Contexto 9/11 (2005): 71-84.

Murra, John V. "El 'archipiélago vertical' once años después". El mundo andino. Población, medio ambiente y economía. Lima: Pontificia Universidad Católica del Perú, 2002. 132-39.

"El control vertical de un máximo de pisos ecológicos en la economía de las sociedades andinas". Visita de la provincia de León de Huánuco en 1562. Iñigo Ortiz de Zúñiga, Visitador, vol. 2. Huánuco: Universidad Nacional Hermilio Valdizán, 1972. 427-76.

The Economic Organization of the Inca State. Chicago: University of Chicago, 1956.

"Los límites y las limitaciones del 'Archipielago vertical' en los Andes". Maguaré 1/1 (1981): 93-98.

"Studies in Family Organization in the French Caribbean." Transaction of the New York Academy of Science 19 (1957): 372-78.

Nettleford, Rex. Caribbean Cultural Identity. The Case of Jamaica: An Essay in Cultural Dynamics. Los Angeles: University of California, 1979.

Noriega Bernuy, Julio E. Caminan los Apus: escritura andina en migración. Lima/ Galesburg: Pakarina Ediciones/Knox College, 2012.

Platt, Tristan. "'Desde la perspectiva de la isla'. Guerra y transformación en un archipiélago vertical andino: Macha (Norte de Potosí, Bolivia)". Chungara. Revista de Antropología Chilena 42 (2010): 297-324.

Revista Iberoamericana, Vol. LXXXI, Núm. 253, Octubre-Diciembre 2015, 973-985 
Ribeiro, Darcy. Las Américas y la civilización: proceso de formación y causas del desarrollo desigual de los pueblos americanos. Prólogo, María Elena Rodríguez Ozán; cronología y bibliografía, Mercio Pereira Gomes. Caracas: Biblioteca Ayacucho, 1992.

Vertovec, Steven. "Conceiving and Researching Transnationalism". Ethnic and Racial Studies 22/2 (1999): 447-62.

Zevallos Aguilar, Ulises Juan. "Desplazamiento y transnacionalismo en la construcción de una identidad cultural andina en los EE.UU." Perspectivas Latinoamericanas 1 (2004): 158-73.

"Hacia una topografía del archipiélago cultural andino". Socialismo y participación 87 (2000): 101-10. 
